\title{
Diversity Properties of Multiantenna Small Handheld Terminals
}

\author{
Wim A. Th. Kotterman \\ Department of Communication Technology (KOM), Aalborg University, 9220 Aalborg Ø, Denmark
}

Email:wim@kom.aau.dk

Gert F. Pedersen

Department of Communication Technology (KOM), Aalborg University, 9220 Aalborg Ø, Denmark

Email: gfp@kom.aau.dk

\author{
Kim Olesen \\ Department of Communication Technology (KOM), Aalborg University, 9220 Aalborg Ø, Denmark \\ Email:ko@kom.aau.dk
}

Received 23 June 2003; Revised 26 February 2004

\begin{abstract}
Experimental data are presented on the viability of multiple antennas on small mobile handsets, based on extensive measurement campaigns at $2.14 \mathrm{GHz}$ with multiple base stations, indoors, from outdoor to indoor, and outdoors. The results show medium to low correlation values between antenna branch signals despite small antenna separations down to $0.16 \lambda$. Amplitude distributions are mainly Rayleigh-like, but for early and late components steeper than Rayleigh. Test users handling the measurement handset caused larger delay spread, increased the variability of the channel, and induced rather large mean branch power differences of up to $10 \mathrm{~dB}$. Positioning of multiple antennas on small terminals should therefore be done with care. The indoor channels were essentially flat fading within $7 \mathrm{MHz}$ bandwidth $(-6 \mathrm{~dB})$; the outdoor-to-indoor and outdoor channels, measured with $10 \mathrm{MHz}$ bandwidth, were not. For outdoor-to-indoor and outdoor channels, we found that different taps in the same impulse response are uncorrelated.
\end{abstract}

Keywords and phrases: mobile radio channel, small multiantenna devices, measurement analysis, branch correlation, Doppler spectrum, user influence.

\section{INTRODUCTION}

Research on smart antennas or smart algorithms seem to have focused on base stations (BSs) and fixed terminals with relatively little research being devoted to the benefits of multiple antennas on small mobile terminals. A reason for this surely must be the still frequently expressed opinion that a separation between antennas of at least half a wavelength is needed to get branch correlation coefficients under a threshold of 0.7 needed for exploiting the diversity potential. In this context, one often quotes Jakes [1], but he considered amplitude correlation coefficients for early narrowband mobile systems, whereas for GSM-like systems, it was shown that at least for some forms of diversity, such a threshold does not exist. Diversity gain then increases continuously with decreasing correlation [2]. Moreover, Vaughan and Andersen [3] showed that in the ideal case, the antenna patterns are orthogonal with respect to the incoming wave field, which theoretically can be achieved even at zero separation for par- ticular environments. This of course implies that the achievable diversity gain depends on both the antenna design and the specific propagation environment. In this respect, spatial separation is merely a factor in decorrelation between antenna signals as are polarisation properties. Experimental confirmation has been documented from the early 1990's onwards $[4,5,6,7]$. Please note that the overriding importance of handset antennas being small, while efficient and wideband, leaves little room for engineering radiation patterns.

In the framework of a project on smart antennas for small handsets at Aalborg University (AAU), three measurement campaigns were organised in different propagation environments with and without users, as users have a strong influence on the reception by handheld terminals $[8,9]$. During these campaigns, we used our proprietary measurement system [10] with our "optical" handset without conducting cables, but using signal transport by optic fibre instead [11]. This paper reports on the findings, with some emphasis placed on the three classical quantities 

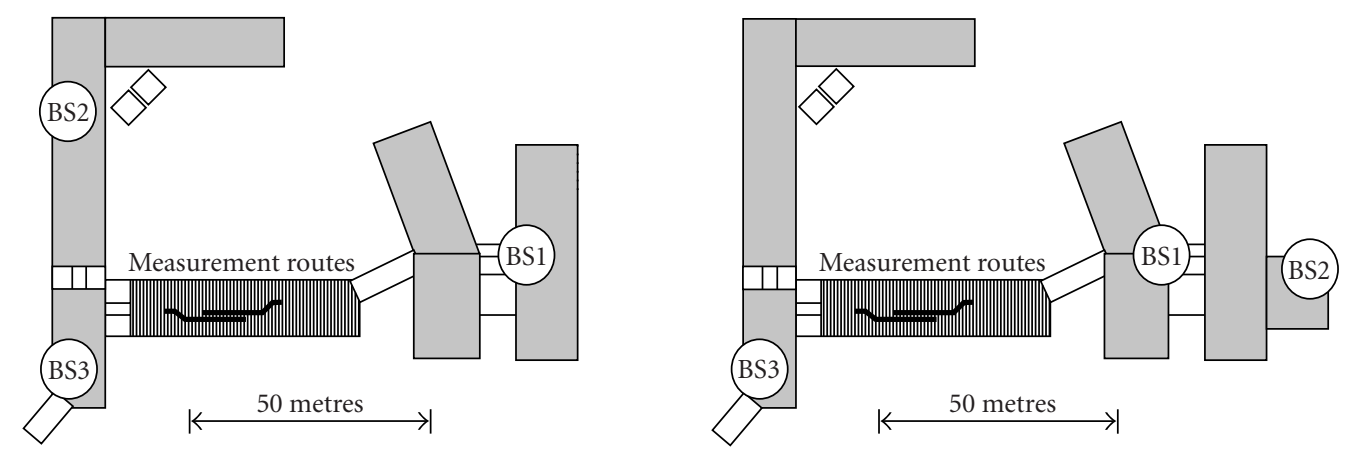

(a)
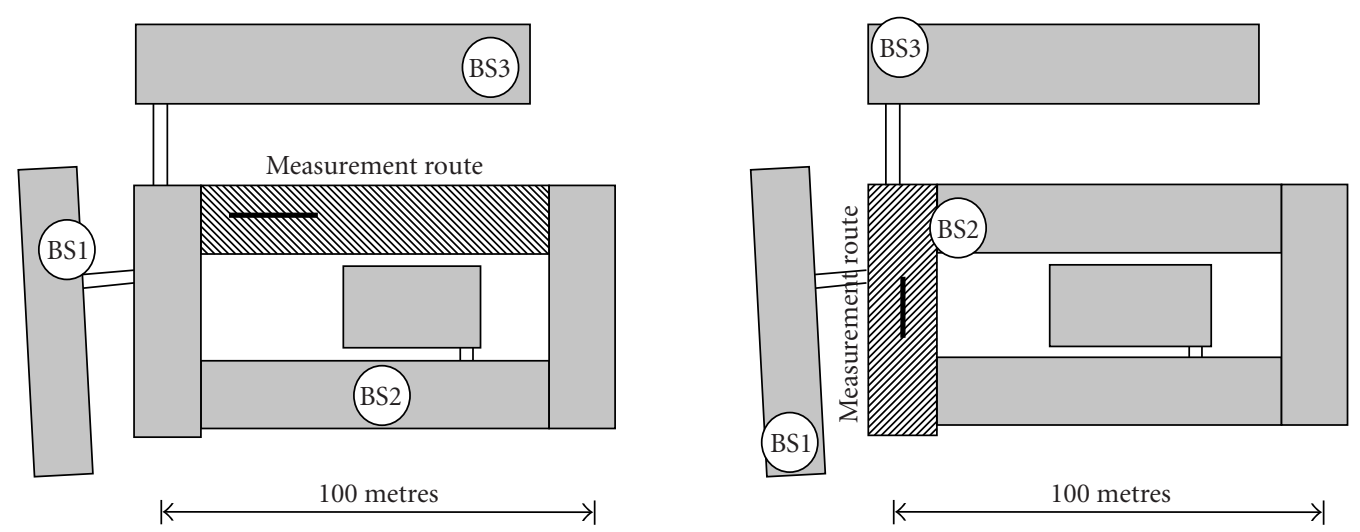

(b)

FIGURE 1: Measurement situations and BS configurations for the indoor campaign: (a) star configuration for the new building (left) and inline configuration for the new building (right); (b) star configuration for the old building (left) and inline configuration for the old building (right). The new buildings' first route is to the right, walked from left to right, while the second route is to the left, walked from right to left.

determining diversity gain: branch correlation coefficients, amplitude distributions, and (mean) branch power differences [12]. The structure of this paper is as follows: first, the measurement setup is discussed with the chosen scenarios, the use of test users, and the equipment. Next, the processing of the data is described, followed by results and discussion. Conclusions form the last section.

\section{MEASUREMENT SETUP}

The measurement campaigns should provide realistic data for channel models to be used for research into smart antennas for small handsets. Therefore, the data should be gathered in a way that reflects typical use of handheld devices and typical handheld devices themselves, including size, antenna types, and locations of major components like display, keypad, and antennas. This means measuring in different cellular scenarios, with users handling the terminal in different ways. Some aspects of the choices made for the campaigns will be treated in the next sections.

\subsection{Cellular scenarios}

Three cellular scenarios were chosen: indoor, outdoor-toindoor, and outdoor.

For the indoor campaigns, we selected two different buildings as the type of construction determines the propagation regime. One is the university building in downtown Aalborg as example of the early twentieth-century building style: heavy walls with single-sheet windowpanes, favouring penetration through the windows with only limited guiding in corridors. As for the second building, a modern office building at the campus was selected, having a reinforced concrete structure with plasterboard partitioning and metalcoated windows as in Figure 1. Little penetration from outside should be expected as most signals are guided inside.

For the outdoor-to-indoor campaigns, the old university building was selected. In this campaign, the link budget was improved, which allowed placing BSs at more distant and more obstructed locations as in Figure 2. Free-in-air measurements were added too, with the handset on a pole without the user as a form of reference. 
(BS1)

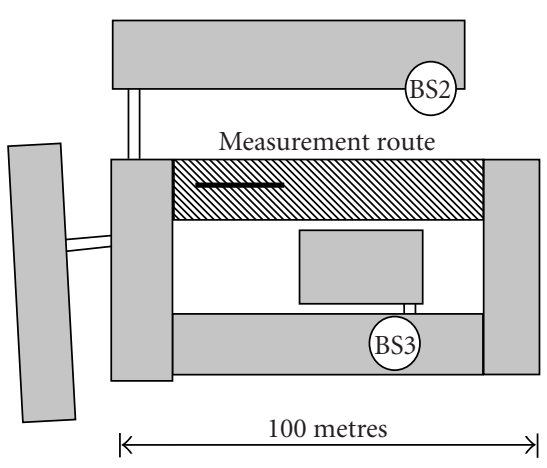

(a)
(BS1)

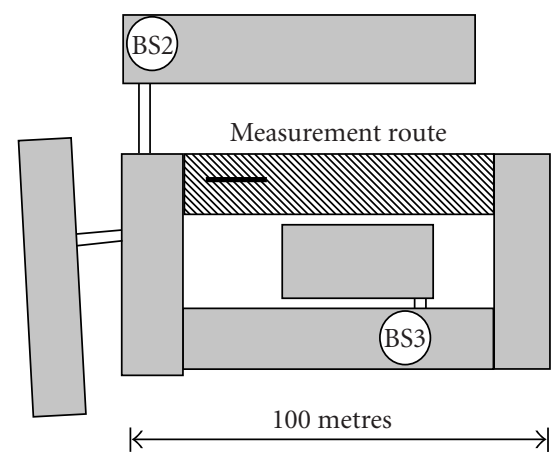

(b)

FIGURE 2: Measurement situations and BS configurations for the outdoor-to-indoor campaign: (a) star configuration for the old building and (b) inline configuration for the old building.

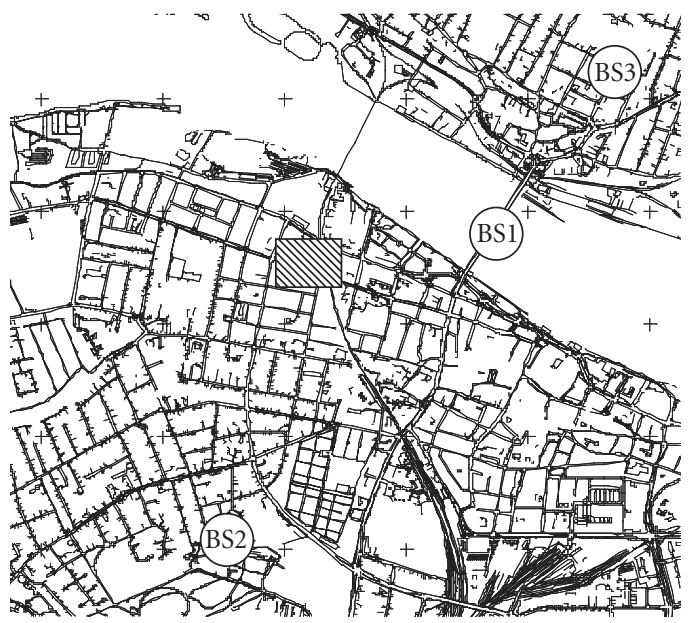

(a)

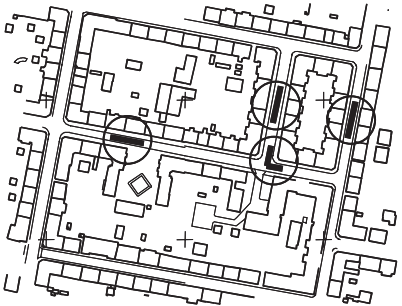

(b)

FIgURe 3: Measurement situation for the outdoor campaign: (a) BSs in the centre of Aalborg with the measurement area shaded (2.75 $\times$ $\left.2.5 \mathrm{~km}^{2}\right)$ and (b) enlarged outdoor measurement area with the four measurement trajectories encircled $\left(245 \times 180 \mathrm{~m}^{2}\right)$.

For the outdoor campaigns, the measurements were aimed at medium size cells in a European downtown area with propagation conditions and path lengths clearly different from the two other environments. Path lengths ranged from 1 to $2 \mathrm{~km}$ as in Figure 3. The area in Aalborg with the smallest ratio of street width to rooftop height was chosen and for link budget reasons, relatively high BSs were employed. Here only results will be shown for the handset tied to a torso phantom in a trailer behind the measurement van due to low signal-to-noise ratio (SNR), with the test users inside the van.

\subsection{Interference situations}

The choice for measuring multiple BSs simultaneously is based on the fact that interference certainly is one of the major aspects of cellular network operation. In CDMA systems, intercell interference may be less important than in TDMA systems, but in CDMA, the best candidate for soft handover/macrodiversity is most likely the strongest interferer.

Two different BS configurations have been chosen, a "star" BS configuration and an "inline" configuration. Figure 1 gives an example of the two configurations for the indoor measurements, and Figure 2 for the outdoor-toindoor measurements. Of the outdoor measurements, represented in Figure 3, only the inline data is used.

The star configuration imitates the conditions at the edge of a cell, with three BSs surrounding the mobile station at comparable distances. This maximises interference levels but the correlation between interfering signals and the desired 


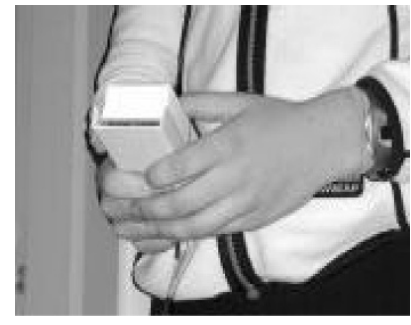

(a)

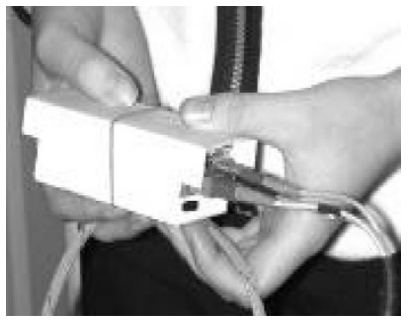

(b)

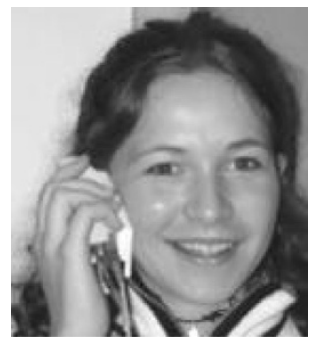

(c)

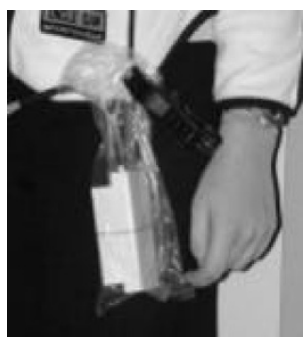

(d)

FIGURE 4: The four ways of handling the handset measurement by a test person. (a) portrait, (b) landscape, (c) at the ear, and (d) at the hip.

signal is most likely low. In the inline configuration, the levels of interference differ but the correlation between the interferers and the serving BS could be higher than in the star configuration, especially under waveguiding conditions.

\subsection{Use of test persons}

The use of a number of test persons is based on the experience that the user has a major impact on handset performance [9], for instance, due to body-induced losses (hand, head), due to orientation of the handset, due to specific movements of the user, and so forth. Therefore, we aimed at having at least ten users run the prescribed test route. The users were also asked to hold the handsets in a number of different ways, at the ear and in the hand in two different ways. For the outdoor-to-indoor campaign, enough link budget was available to incorporate placement at the hip too. Figure 4 gives an impression of the various positions.

The position of the terminal in the hand, called "portrait," imitates the present use of a phone when updating the calendar or SMS directory. The "landscape" mode refers to using the newly developed models with large displays. Carrying the terminal at the hip mainly simulates the idle mode. As mentioned earlier, for the outdoor campaign, only phantom measurement results will be presented.

\subsection{Equipment}

The measurement equipment used was AAU's proprietary equipment [10], based on a correlating receiver, sampling the received signal in $I$ and $Q$ on baseband signal, with correlation of the 511-chips long $m$-sequence in postprocessing. Simultaneous sounding of BSs was achieved in the code domain. Throughout the campaigns, we used our optical handset, in two versions, that truly represents a small receiving device without the radiation pattern disturbing effects of conducting cables [11]. The antennas employed on these handsets were chosen to reflect practical implementations and designed to occupy as small volume as possible for the required bandwidth. This leads to monopole-like antennas that act as matching or coupling to the terminal casing that then acts as the main radiator. In this way, very small antennas can show good efficiency and bandwidth compared to the size of the antenna elements because the casing is the main radiator, not the antenna element itself. However, this approach allows the designer but little control over the antenna radiation patterns and polarisation properties. Also, radiation characteristics are dissimilar for similar antenna elements placed at different positions on the terminal, but this on the other hand contributes significantly to the decorrelation of the antenna signals. We used two different approaches frequently seen with handsets at that time: stubs that are either monopoles or helices, and integrated antennas, in our case planar inverted $\mathrm{F}$ antennas (PIFAs), to see whether this would make a difference.

The first version of the handset was used in the indoor campaign with either two monopoles or PIFAs, seen in Figure 5 a with monopoles. Chassis dimensions are $103 \times 48 \times$ $35 \mathrm{~mm}^{3}(h \times w \times t)$. During the measurements, the wire elements were stabilised with a foam radome. The PIFAs were screwed directly onto the SMA connectors visible at the front. The element size was $0.1 \lambda \times 0.1 \lambda \times 0.05 \lambda(h \times w \times t)$, but due to the use of dielectrics, the free in air size was somewhat smaller, making it possible to have a distance between the antennas of only $0.16 \lambda$ centre to centre. The second version was used in the other two campaigns. For outdoor-toindoor, it was used with both four helices and PIFAs (different from those used indoors) as in Figures $5 b$ and $5 c$. For the outdoor campaign, the second handset was only equipped with four small (dielectric) PIFAs as in Figure 5d. The first change of antennas was mainly motivated by the mechanic vulnerability of the antenna elements and the wish to have a smooth surface for the second handset. The open PIFA structures used for outdoor-to-indoor proved to be vulnerable too. Consequently, solid dielectric PIFAs were used for the outdoor campaign. Chassis dimensions of the second handset are $92 \times 51 \times 37 \mathrm{~mm}^{3}(h \times w \times t)$. For protection of the antennas, this handset was used with a plastic lid, visible in Figure 4. The antennas of the second handset have all been measured in the anechoic chamber, spherically, and dualpolarised. Figure 6 shows an example of the radiation patterns for the top two PIFAs antennas in Figure $5 \mathrm{c}$ used in the outdoor-to-indoor campaign. For reasons of clarity and due to the limited space, only one plane cut is shown, normal to the faceplate and parallel to the length axis of the handset. Although the patterns are quite similar to each other in both polarisations, the achievable decorrelations are substantial as will be shown in Section 4. Those decorrelations result 


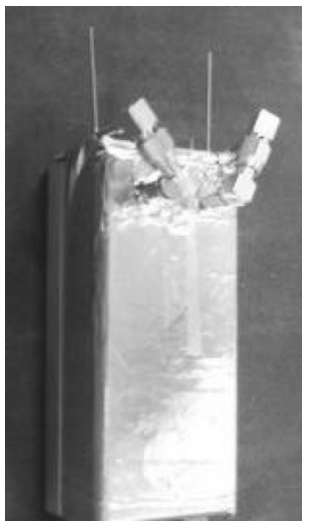

(a)

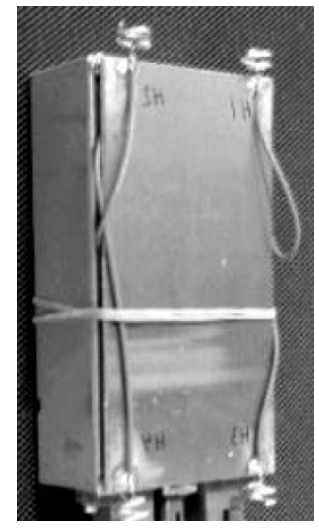

(b)

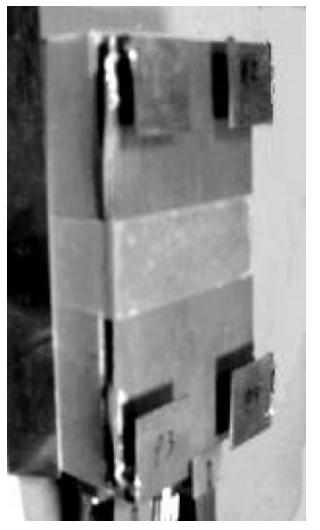

(c)

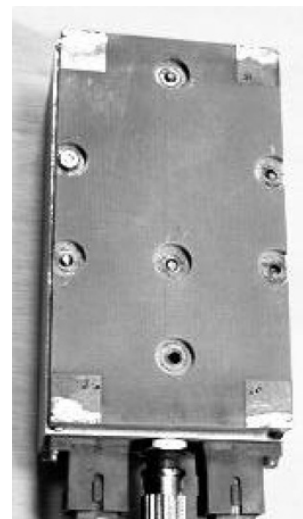

(d)

FIGURE 5: Antenna placements on handsets: (a) first handset with monopoles for indoor; (b and c) second handset with helices and PIFAs for outdoor-to-indoor; and (d) second handset with dielectric PIFAs for outdoor. All handsets are shown without radome or protective cover.

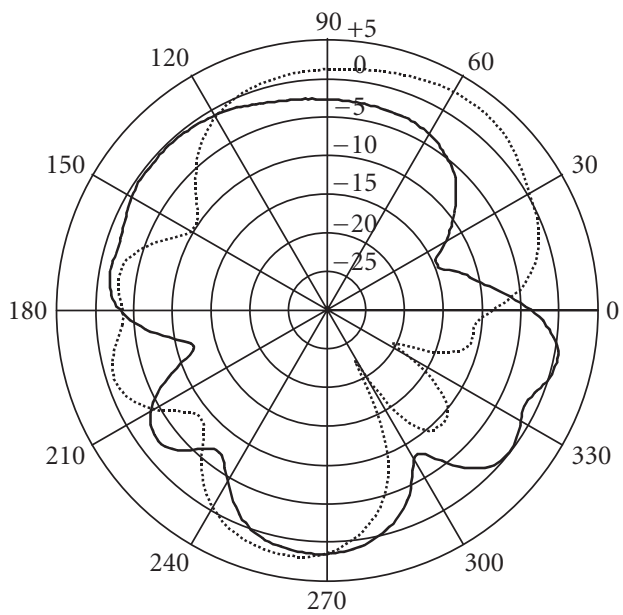

$-E_{\phi}$
$\cdots \cdots \cdots$
$E_{\theta}$

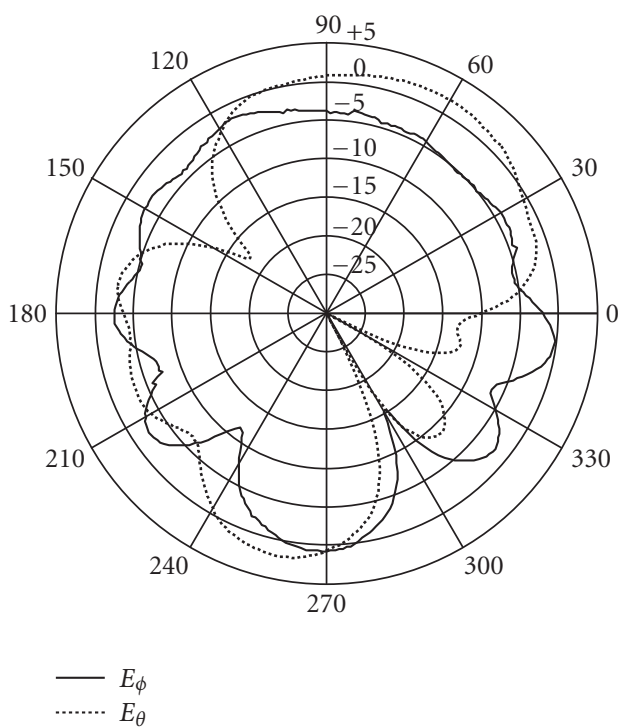

(b)

FiguRE 6: Measured radiation patterns (copolar and cross-polar) for two of the PIFAs in the outdoor-to-indoor campaign (Figure 5c) in the plane perpendicular to the faceplate and parallel to the length axis of the terminal: (a) top left antenna and (b) top right antenna. The amplitudes along the radial are in $\mathrm{dB}$.

from the projection of the angular distributions of the incoming wave field onto the radiation patterns (in both polarisations) of the antennas, (see Vaughan [3]). However, seeing the similarities of the antenna patterns, detailed knowledge is required of the angular distributions of the incoming wave field when analysing antenna performance. We did not measure such angular distributions for the environments in these campaigns and considered that to be out of scope for these investigations too. Consequently, we will not expand on the performance of specific antenna types.
Due to a different chip rate, the effective bandwidth was $7 \mathrm{MHz}(-6 \mathrm{~dB})$ for indoor campaign and $10 \mathrm{MHz}$ for outdoor-to-indoor and outdoor campaigns. For indoor and outdoor-to-indoor campaigns, the impulse response acquisition was triggered equidistantly in time, and for the outdoor one, equidistantly in distance. All these changes in the equipment resulted from insight gained during the campaigns, spanning more than a year. The main system parameters of the sounding equipment for the different campaigns are summarised in Table 1. 
TABLE 1: Main parameters of channel sounding equipment used in the different campaigns.

\begin{tabular}{llll}
\hline System parameter & Indoor & Outdoor-to-indoor & Outdoor \\
\hline PN code length & 511 & 511 & 511 \\
PN code chip rate $(\mathrm{MHz})$ & 3.8325 & 7.665 & 7.665 \\
Bandwidth $(-6 \mathrm{~dB})(\mathrm{MHz})$ & 7 & 10 & 10 \\
Baseband sampling $(\mathrm{MHz})$ & 15.36 & 15.36 & 15.36 \\
IR acquisition rate & $30\left(\mathrm{~s}^{-1}\right)$ & $25\left(\mathrm{~s}^{-1}\right)$ & $1 / 0.0544\left(\mathrm{~m}^{-1}\right)$ \\
Carrier frequency $(\mathrm{MHz})$ & 2140 & 2140 & 2140 \\
Optical handset type & No. 1 & No. 2 & No. 2 \\
Number of HS antennas & 2 & 4 & 4 \\
Antenna types (separation) & Monopoles $(0.29 \lambda)$ & Helices $(0.21 \lambda / 0.51 \lambda)$ & PIFAs $(0.21 \lambda / 0.51 \lambda)$ \\
\hline
\end{tabular}

\section{DATA PROCESSING}

The purpose of the measurements is to provide data for tapped delay line models. Therefore, the data processing should render suitable tap delays and find the characteristics per tap signal over time or distance measurement. Relations between tap signals should be established too. The characteristics considered are

(1) amplitude/power distribution per tap;

(2) cross-correlation between fading patterns of antenna branch signals per tap;

(3) mean branch power differences;

(4) Doppler spectrum per tap;

(5) cross-correlation between fading patterns of BS signals for the same tap and antenna branch;

(6) cross-correlation between fading patterns of tap signals for the same antenna branch.

The first three are the classical parameters determining diversity gain: the Doppler spectrum determines the evolution of tap signals over time/distance, the cross-correlation between tap signals could influence equalising strategies, and cross-correlation between BSs or interferers influences the gain by both antenna and macrodiversity [13, 14, 15]. The amplitude distribution has also implications on the coverage and the outage performance of system cells; see, for example, $[16,17,18]$. The indoor measurements were essentially flat fading, so only a single tap was used. For the outdoor measurements, no total signal power was computed, so no mean branch power differences were derived.

\subsection{General preprocessing}

Directly after every campaign, the full set of measurement equipment is taken into a shielded room and calibrated back to back, using attenuators and coaxial cables instead of antennas. The measured data is scaled with the calibration data and correlated with the back-to-back system responses.

\subsubsection{Processing specifics for indoor}

The indoor responses were essentially single tap. Therefore, the processing consisted of determining the tap delay per BS and per antenna branch and of separation of slow and fast fading signals from the extracted tap signal. The purpose of using these fading types is to connect to existing modelling schemes in which the fading is modelled as the product of a slow fading term and a fast fading term instead of modelling Nakagami distributions.

The tap excess delay $\tau_{m}$ of the single tap was determined per measurement run from the power over all impulse responses $h\left(\tau, t_{i}\right)$ as $\tau_{m}=\operatorname{argmax}_{\tau}\left\{\left|h\left(\tau, t_{i}\right)\right|^{2}\right\}$, with $t_{i} \in\left\{t_{1}, \ldots, t_{512}\right\}$ the measurement instance. The slow fading power $p_{\text {slow }}$ was defined as the lowpass filtered output of the received power $\left|h\left(\tau_{m}, t_{i}\right)\right|^{2}$ at delay $\tau_{m}$, by convolution with a real-valued Hanning window $W_{H}$ of length 48:

$$
p_{\text {slow }}\left(t_{i}\right)\left|h\left(\tau_{m}, t_{i}\right)\right|^{2} \otimes W_{H}\left(t_{i}\right)
$$

with $W_{H}(k)=0.5-0.05 \cdot \cos (2 \pi \cdot k / 48) ; k \in\{1, \ldots, 48\}$. The length of the Hanning window was not critical, but the length of 48 rendered fast fading signals that matched Rayleigh distributions quite well, corresponding to 1.6 seconds or a few metres at walking speed. The complex fast fading signal $h_{\text {fast }}$ is the complex received signal divided by the square root of the slow fading power:

$$
h_{\text {fast }}\left(t_{i}\right)=\frac{h\left(\tau_{m}, t_{i}\right)}{\sqrt{p_{\text {slow }}\left(t_{i}\right)}} .
$$

Further processing is done on both the fast fading signal and the (square root of the) slow fading power.

\subsubsection{Processing specifics for outdoor and outdoor-to-indoor cases}

For the outdoor and outdoor-to-indoor measurement results, tap delays and tap signal characteristics were extracted by using a two-dimensional SAGE algorithm [19]. Based on the rendered estimates, the tap signals (over time for the outdoor-to-indoor case and over distance for the outdoor one) were constructed as described in [20]. The tappeddelay line structure is determined by the BS, so it is the same for the different antenna branches and users. This means that each antenna branch and each user signal has the same tap 
delays for the response to a particular BS on a particular measurement location, only differing from other branches/users in complex amplitude and Doppler values. For these tap signals, no fast or slow fading signals were extracted. The SAGE estimation process operated on twenty consecutive impulse responses at a time, with the next estimation cycle half overlapping the former. Not always were the estimates available for every tap delay, so on certain measurement intervals, gaps occurred in the constructed tap signals, making the interpretation of slow and fast fading very hard.

\subsection{Power distributions}

Power distributions were derived for indoor data for both the fast and the slow fading power. For outdoor and outdoor-toindoor data, power distributions were derived for the power in individual tap signals under the constraint that for at least $25 \%$ of the tap signal duration, SAGE estimates were available. Data were pooled over measurement runs before determining cumulative distribution functions (CDFs).

\subsection{Antenna branch correlations}

For indoor data, antenna branch correlations for the same BS were determined for both fast and slow fading for the two antenna branches. For outdoor and outdoor-to-indoor data, correlations between each of the six combinations of two out of the four antenna branches were determined for each tap. The correlation per tap was performed over those points where both branches in a combination had (constructed) signal under two constraints: the first being that the tap signal in both branches should have a mean power higher than $-12 \mathrm{~dB}$ below the highest mean tap power for the respective branch, and the second that the number of common points was larger than 127 ( $25 \%$ of the tap signal duration). The mean power threshold was imposed because of the observed increasing inaccuracy of the SAGE algorithm with decreasing tap powers.

All correlations are complex correlations between variations around the mean. The values given are mean and standard deviation of the magnitude of the correlation coefficients, pooled over users/measurement runs, antenna types, use positions (if applicable), BSs, BS configurations, and antenna branch combinations (for outdoor and outdoor-toindoor cases).

\subsection{Mean branch power differences}

The mean branch power difference was determined as the difference in the mean power received per branch from a single BS over a single measurement run. For the indoor case, this was the difference in mean values of the slow fading power per antenna branch (fast fading power has mean 1). For the outdoor-to-indoor case, the impulse response powers were integrated over the impulse response duration. For each measurement run, this total received power was averaged per antenna branch. The mean branch power difference per measurement run for each of the six combinations of two out of the four antenna branches was the difference in the respective average total received powers. For the outdoor case, no mean branch power differences were determined as the computation of the total received power was too sensitive to the influence of noise on the integration interval. As the actual values were often uniformly spread over a large interval symmetric around zero, the mean and standard deviations are given for the absolute values of the differences. The values are pooled over measurement runs, antenna types, use positions, BSs, BS configurations, and antenna branch combinations (for outdoor-to-indoor case).

\subsection{Doppler spectra}

Doppler spectra were made up per measurement run over the full length of each tap signal. For the indoor case, the fast fading signal was used. For plotting purposes, the individual spectra were added powerwise (over measurement runs). The presented results in Table 2 are the average values and the standard deviation of the absolute value of the mean Doppler shift and the Doppler spread determined for each individual spectrum after pooling over users/measurement runs, antenna types, use positions (if applicable), BSs, BS configurations, antenna branches, and taps. Results from tap signals with a mean power lower than $-12 \mathrm{~dB}$ below the highest mean tap power for the respective branch were discarded. For comparison, the shifts and spreads are normalised with respect to the Nyquist rate of the impulse response acquisition, $15 \mathrm{~Hz}$ in the indoor case, $12.5 \mathrm{~Hz}$ in the outdoor-to-indoor case, and $9.2 \mathrm{~m}^{-1}$ in the outdoor case.

\subsection{Interferer correlation}

Interferer correlation was defined as the correlation between two BS signals received on the same antenna branch for a single measurement run. For the indoor case, these (complex) correlations were determined for both antenna branches for all three combinations of two out of three BSs, for both the fast and slow fading signals. For the outdoor-to-indoor case, these correlations have been derived from the total received power. As the power still showed fading in this scenario, the slow fading power was extracted from the total received power by the same smoothing operation as in (1). The fast fading power was defined as the total received power divided by the slow fading power. The interferer correlation was determined as the correlation between either the slow or fast fading powers for all three combinations of two out of three BSs, for all four antenna branches separately. The correlation is of the covariance type. No interferer correlation was determined for the outdoor case. The values given are mean and standard deviation of the absolute value of the correlation coefficients, pooled over measurement runs, antenna types, use positions, BS configurations, antenna branches, and BS combinations.

\subsection{Intertap correlations}

Intertap correlations are the complex correlations between fading patterns of the same tap of the same BS signal on two antenna branches, determined per measurement run. For outdoor and outdoor-to-indoor cases, these correlations were computed for each of the possible combinations (no 
TABLE 2: Results of data processing for the different measurement campaigns. Given are the averages of the magnitudes of the considered variable, with standard deviations of the magnitudes in parentheses.

\begin{tabular}{|c|c|c|c|c|c|c|}
\hline \multicolumn{2}{|c|}{ Channel characteristic } & $\begin{array}{l}\text { Indoor new } \\
\text { building }\end{array}$ & $\begin{array}{l}\text { Indoor old } \\
\text { building }\end{array}$ & $\begin{array}{l}\text { Outdoor-to-indoor } \\
\text { trolley }\end{array}$ & $\begin{array}{l}\text { Outdoor-to-indoor } \\
\text { test persons }\end{array}$ & Outdoor \\
\hline \multirow{2}{*}{$\begin{array}{l}\text { Amplitude } \\
\text { distributions }\end{array}$} & $\begin{array}{l}\text { Fast } \\
\text { fading }\end{array}$ & & & \multirow{2}{*}{$\begin{array}{l}\text { Mainly } \\
\text { Rayleigh }\end{array}$} & \multirow{2}{*}{$\begin{array}{l}\text { Mainly } \\
\text { Rayleigh }\end{array}$} & \multirow{2}{*}{$\begin{array}{l}\text { Mainly } \\
\text { Rayleigh }\end{array}$} \\
\hline & $\begin{array}{l}\text { Slow } \\
\text { fading }\end{array}$ & $\begin{array}{l}\text { Lognormal } \\
(\sigma \sim 3-7 \mathrm{~dB})\end{array}$ & $\begin{array}{l}\text { Lognormal } \\
(\sigma \sim 3-7 \mathrm{~dB})\end{array}$ & & & \\
\hline \multirow{2}{*}{$\begin{array}{l}\text { Branch } \\
\text { correlations }\end{array}$} & $\begin{array}{l}\text { Fast } \\
\text { fading }\end{array}$ & $0.48(0.26)$ & $0.53(0.24)$ & \multirow{2}{*}{$0.33(0.15)$} & \multirow{2}{*}{$0.32(0.16)$} & \multirow[b]{2}{*}{$0.42(0.23)$} \\
\hline & $\begin{array}{l}\text { Slow } \\
\text { fading }\end{array}$ & $0.82(0.16)$ & $0.77(0.18)$ & & & \\
\hline \multicolumn{2}{|c|}{$\begin{array}{l}\text { Mean branch power } \\
\text { differences }(\mathrm{dB})\end{array}$} & $2.2(1.5)$ & $1.8(1.2)$ & $2.3(1.6)$ & $4.4(3.0)$ & Not determined \\
\hline \multirow{2}{*}{ Doppler } & Mean $^{\ddagger}$ & $0.25(0.15)$ & $0.23(0.17)$ & $0.22(0.16)$ & $0.41(0.22)$ & $0.52(0.29)$ \\
\hline & Spread $^{\ddagger}$ & $0.59(0.10)$ & $0.67(0.14)$ & $0.43(0.07)$ & $0.45(0.10)$ & $0.34(0.21)$ \\
\hline \multirow{2}{*}{$\begin{array}{l}\text { Interferer } \\
\text { correlation }\end{array}$} & $\begin{array}{l}\text { Fast } \\
\text { fading }\end{array}$ & $0.14(0.12)$ & $0.08(0.05)$ & $0.05(0.05)^{\dagger}$ & $0.05(0.05)^{\dagger}$ & \multirow[b]{2}{*}{ Not determined } \\
\hline & $\begin{array}{l}\text { Slow } \\
\text { fading }\end{array}$ & $0.60(0.23)$ & $0.42(0.23)$ & $0.31(0.20)^{\dagger}$ & $0.29(0.20)^{\dagger}$ & \\
\hline \multicolumn{2}{|l|}{$\begin{array}{l}\text { Intertap } \\
\text { correlations }\end{array}$} & N.A. & N.A. & $0.19(0.12)$ & $0.23(0.14)$ & $0.08(0.10)$ \\
\hline
\end{tabular}

‡ Values in fractions of Nyquist rate, determined by snapshot repetition rate.

${ }^{\dagger}$ Based on total received power, not on complex signal.

permutations) of two out of all tap signals for a given antenna branch and BS under two constraints: the first being that each tap signal should have a mean power higher than $-12 \mathrm{~dB}$ below the highest mean tap power for the branch and the second that the tap signals should have at least 127 points in common. For the indoor case with essentially single-tap channels, no intertap correlations were computed. The values given are mean and standard deviation of the magnitude of the correlation coefficients, pooled over measurement runs, antenna types, use positions (if applicable), BSs, BS configurations, antenna branches, and tap combinations.

\section{RESULTS AND DISCUSSION}

The results of the data processing are summarised in Table 2 . These results will be discussed in more detail in the following sections.

\subsection{Power delay profiles}

The indoor power delay profiles were the shortest; within the measurement bandwidth, they were factually single tap as mentioned. The tap extraction by the SAGE algorithm rendered two to four taps for the outdoor-to-indoor channels with the largest delay spreads for the outside BS, about 80 nanoseconds. The two other BSs showed delay spreads of around 60 nanoseconds. Differences in use positions or antenna types had no large influence on the spreads or the shape of the power delay profiles. For the outdoors case, widely different results were found from almost single-tap channels to 14-tap channels, with the last number maybe limited by the fact that the SAGE extraction gave 15 estimates at a time. The effect of test users seen in the outdoor-toindoor campaign is that users' responses tend to larger delay spread, and so more taps. Also, the variations between responses make it difficult to cluster data from the SAGE algorithm and to arrive at a common tapped-delay representation, especially in cases where the head or body blocks paths to a BS. Therefore, the data for test users of outdoorto-indoor in Table 2 are for the data terminal portrait use position for BS1 and BS3 only in the star configuration.

\subsection{Amplitude distributions}

The amplitude/power distributions that were found are rather classical. For the indoor campaign, the fast fading showed Rayleigh distributions, while the slow fading power was more or less lognormally distributed. The short measurement runs probably did not allow registering a fully developed slow fading pattern. In the star BS configuration, one BS showed a slow fading pattern with a standard deviation of 6-7 dB, while the other two showed rather low values of $3-4 \mathrm{~dB}$. In the inline configuration, two BSs showed higher standard deviations. For outdoor and outdoor-to-indoor cases, the strongest tap signals were Rayleigh distributed, with the weaker taps before or after strong taps showing some Ricean behaviour; see Figure 7 for a typical example.

Outdoor weak taps could show Ricean distributions with strong dominant components but we are not sure how to interpret this. One explanation is that, for these cases almost always, the very small Doppler spread, and therefore the very slow fading pattern [21], did not allow us to measure a fully developed fading pattern over the measurement 


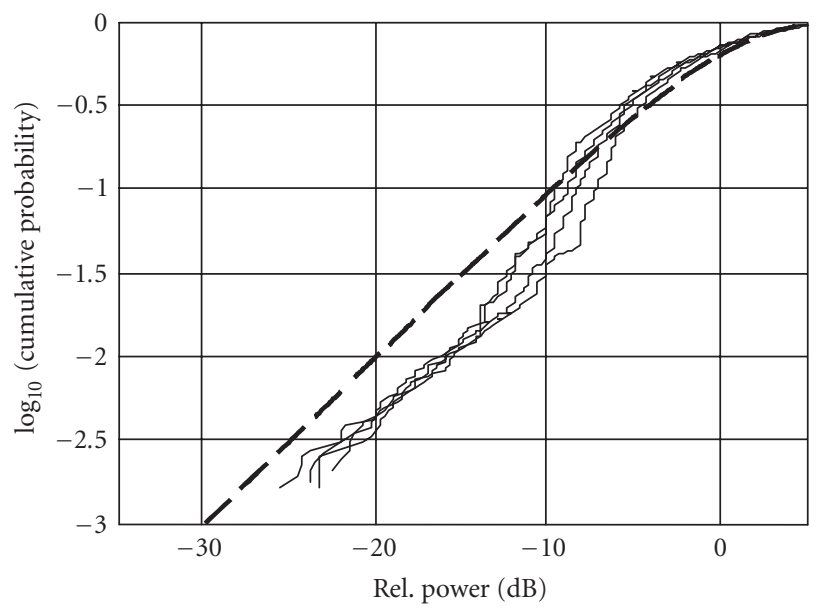

(a)

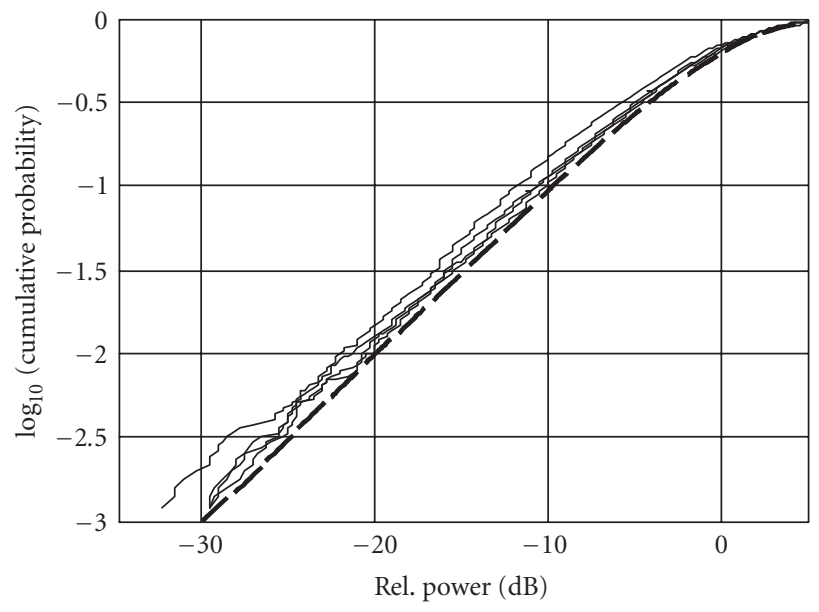

(b)

Figure 7: Comparison between CDFs of (a) a weak early tap (first tap BS3, average power $=-9.8 \mathrm{~dB}$ ) and (b) a stronger next tap (second tap BS3, excess delay = 73 nanoseconds, average power $=-1.7 \mathrm{~dB}$ ) in the outdoor campaign. Dashed lines indicate CDF of power of Rayleigh distributed process.

TABLE 3: Indoor antenna branch correlations for diverse situations. Given are the averages of the magnitudes of the complex correlation coefficients, standard deviations of the magnitudes in parentheses.

\begin{tabular}{|c|c|c|c|c|c|}
\hline \multirow[b]{2}{*}{ Fading type } & \multirow[b]{2}{*}{ Building type } & \multicolumn{2}{|c|}{ BSs in star } & \multicolumn{2}{|c|}{ BSs inline } \\
\hline & & $\begin{array}{c}\text { Monopole } \\
\text { antennas }\end{array}$ & PIFAs & $\begin{array}{c}\text { Monopole } \\
\text { antennas }\end{array}$ & PIFAs \\
\hline \multirow{2}{*}{ Fast fading } & New & $0.33(0.16)$ & $0.70(0.19)$ & $0.32(0.17)$ & $0.48(0.26)$ \\
\hline & Old & $0.39(0.17)$ & $0.70(0.16)$ & $0.33(0.17)$ & $0.72(0.17)$ \\
\hline \multirow{2}{*}{ Slow fading } & New & $0.80(0.16)$ & $0.88(0.15)$ & $0.80(0.16)$ & $0.79(0.18)$ \\
\hline & Old & $0.72(0.18)$ & $0.80(0.13)$ & $0.74(0.19)$ & $0.81(0.18)$ \\
\hline
\end{tabular}

run. Another reason is that the cut-off criterion of $-30 \mathrm{~dB}$ for the SAGE extraction "cuts the tail" of the distribution of weak components.

\subsection{Antenna branch signals correlations}

As regards the antenna branch correlations, Table 2 shows that differences were found between slow and fast fading. Besides, for the fast fading in the indoor case, apparent differences were found between the antenna types. Table 3 illustrates this fact. The monopole antennas show low correlations for fast fading throughout, of about 0.35 on average. The values for the PIFAs are appreciably higher, on average around 0.75 but at a separation of only $0.16 \lambda$ compared to $0.29 \lambda$ for the monopoles. We have insufficient data to determine what causes this higher cross-correlation: the smaller separation, narrower antenna patterns, better similarity of patterns, a stronger cross-coupling between antennas, or a combination of these.

The slow fading is clearly stronger correlated than the fast fading, with mean values around 0.8. There was little difference between BS configurations, use positions, and antenna types, be it that the PIFAs still had slightly higher correla- tion values (Table 3). Possible consequences of slow fading correlation coefficients lower than 1 are increased instantaneous branch power differences, as short-term differences in the mean power, even with zero-mean branch power difference, are added to it. As a possible explanation for slow fading not being fully correlated, it has been suggested that it is a coherent propagation effect rather than a result of blocking or shadowing $[22,23]$.

For outdoors, or for the outdoor-to-indoor case, the values for the antenna branch correlation for the same tap are lower than the values seen indoors, with the lowest values recorded for outdoor-to-indoor, probably due to the larger angular/Doppler spread in this scenario. Outliers for the outdoor scenario were recorded in the middle of the short street, where main contributions to the incoming field showed the smallest Doppler spreads, especially for BS3 (see Section 4.5). In this case, average figures were 0.61 for BS2 and 0.81 for BS3. Line-of-sight connections can be excluded in this street.

In the outdoor-to-indoor case, the helix antennas showed magnitudes of correlation values that on average were $80 \%$ of those recorded for the PIFAs, both for free-in-air measurements and with test persons. 


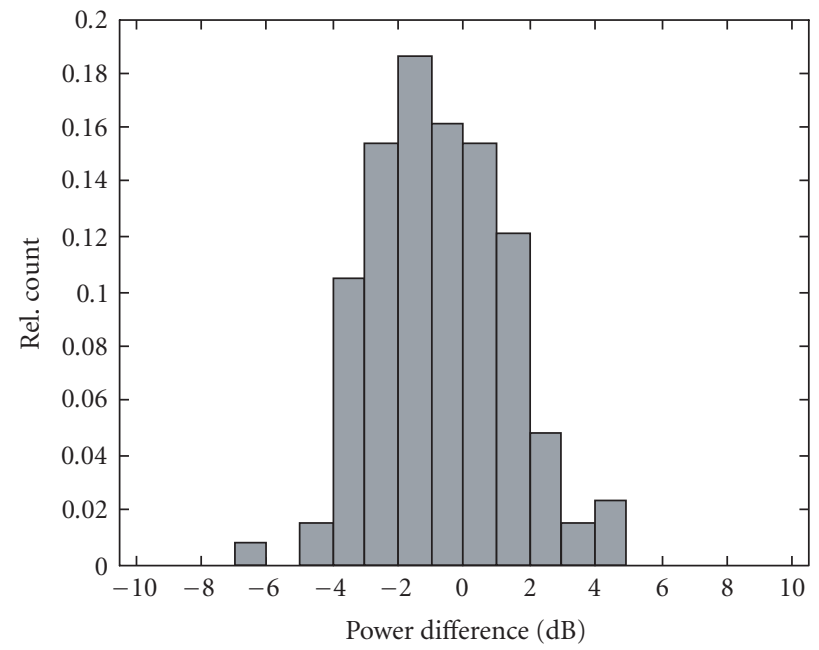

(a)

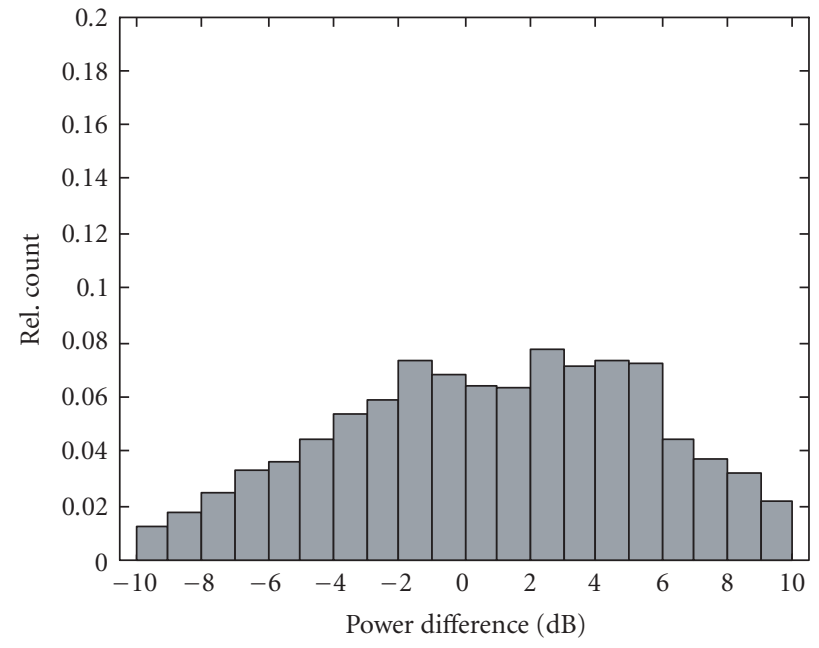

(b)

FIGURE 8: Histograms of mean branch power differences for all measurements in the old building for (a) indoor and (b) outdoor-to-indoor with test persons excluding use position "at the hip."

\subsection{Mean branch power differences}

Mean branch power differences for the outdoor-to-indoor case are quite large, roughly spanning the interval -10 to $+10 \mathrm{~dB}$ (Figure 8), confirming results from others $[8,9]$. However, during the indoor measurements in the same building, lower values were measured of about half that span. We attribute this to the constructional details of the different handsets used in both campaigns. The first handset used indoors has SMA connectors on the face plate, effectively keeping users' fingers away from the ground plane of the monopoles, in this way reducing most of the influences on the radiation efficiency. The dielectric PIFAs used indoors are not so sensitive to proximity effects.

Additionally, the distance between the head and antenna elements could be slightly larger in the first handset. During the outdoor-to-indoor campaign, the handset had a fully smooth surface allowing the user more freedom in handling the phone. The types of antennas used in this campaign could also be more sensitive to proximity effects. In Figure 8, use position "at the hip" is excluded as here much lower values were found, showing more or less the same distribution as the indoor values, as did the free-in-air measurements, again a strong indication that the hands and/or fingers of the users are involved.

Note that the instantaneous branch power differences will be larger than the mean value due to the added effect of (uncorrelated) fast fading and partially uncorrelated slow fading on the branches. The values shown here should be regarded as a conservative estimate.

\subsection{Doppler spectra}

From Table 2, it can be seen that none of the Doppler spectra were symmetric for any of the scenarios. For the indoor environment, the peak in the spectrum was oriented towards the BS, indicating guiding through the corridors (Figure 9a). The ratio of mean Doppler shift and Doppler spread steadily increases when going from the indoor environment, via outdoor to indoor, to outdoor. For the outdoor environment, this means that signal transport is mainly along street orientation, with low angular/Doppler spread. Figure 9a shows an extreme example for a main tap in the mid of the short street. The guiding effects in the corridors of the indoor environment are less pronounced and the differences between the two buildings are in this respect not as large as anticipated. However, the more "open" old building showed a slightly lower mean Doppler shift with higher Doppler spread due to the larger angular spread of the incoming wave fields.

It is not clear why the ratio of the Doppler shift to the Doppler spread has been increased in the old building, from the indoor campaign to the outdoor-to-indoor one. The BS antennas had narrower antenna beam widths in order to increase the link budget, probably at the expense of the angular spread at the measurement spot. Maybe the receiving antennas were more directional too. It could also be that in the outdoor-to-indoor campaign, we managed better to keep the differences in walking speed between the users small.

The differences between PIFAs and helix antennas are on average small and can often be understood from differences in the radiation patterns. For the outdoor-to-indoor case, a seemingly large difference is shown in Figure 10, where the response of the helices on BS2 has a weak first tap, compared to the PIFAs' response. However, as the second tap of the helices' response strongly resembles the PIFAs' first tap, the most likely explanation is that the helices' first tap is the obstructed first arrival of BS2 and is not seen at all by the PIFAs. As we did not record absolute delays, we are not able to check this assumption. 


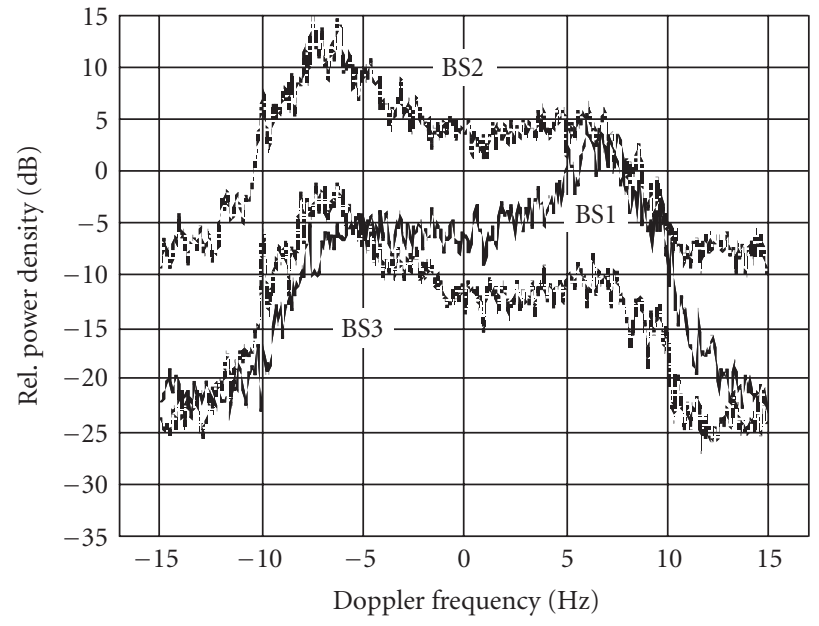

(a)

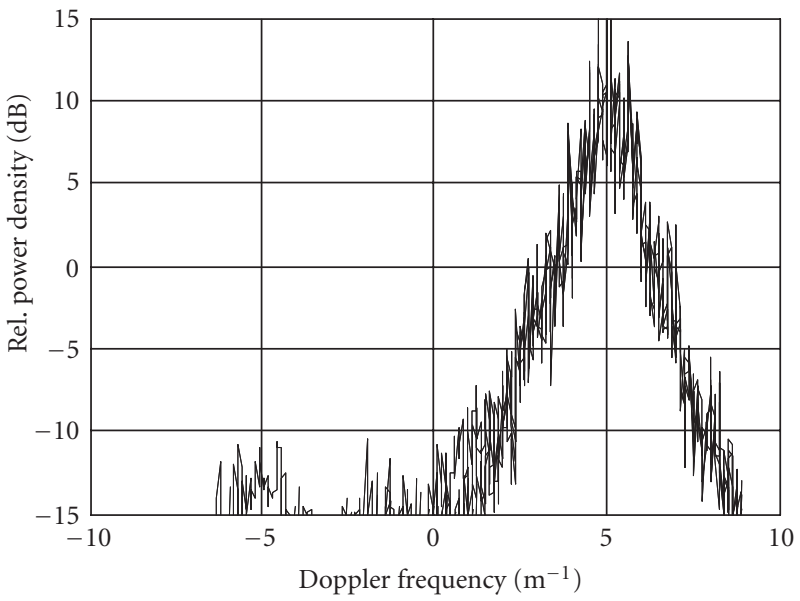

(b)

FIGURE 9: (a) Typical Doppler spectra indoor in the new building: first route, BSs in star, monopole antennas, at the ear (curve BS2 offset by $+10 \mathrm{~dB}$, curve BS3 offset by $-5 \mathrm{~dB}$ ). (b) Highly directive main tap (tap 2) outdoor for BS3 in the middle of the short street (Figure 3).

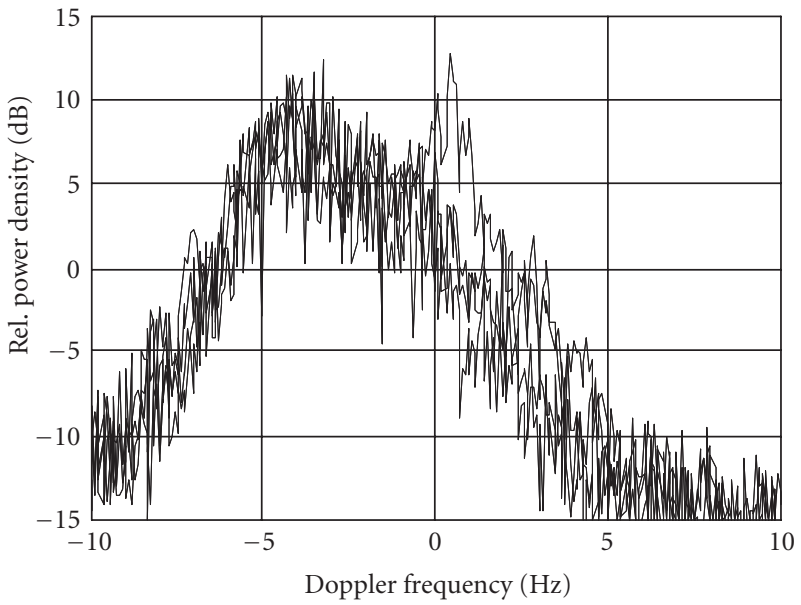

(a)

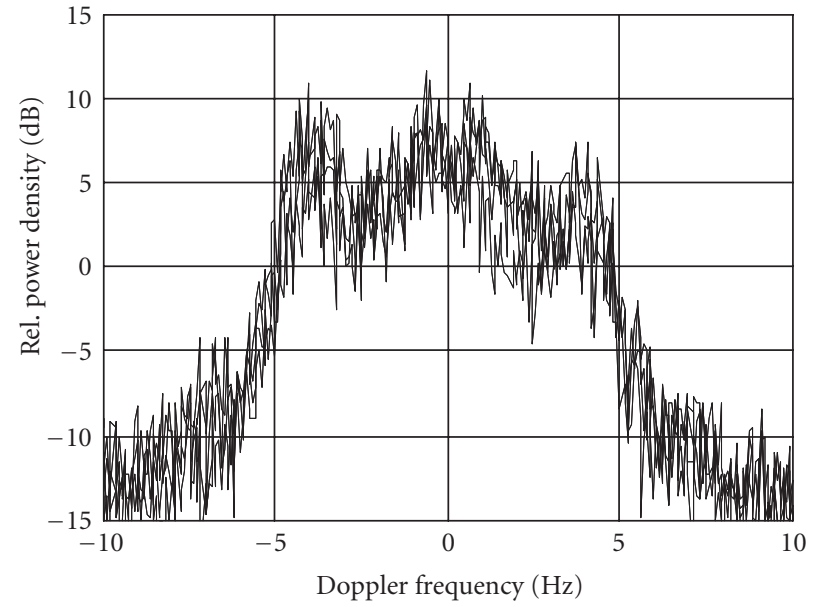

(b)

Figure 10: Average Doppler spectra outdoor-to-indoor for (a) helix ( $\tau_{1}=0$ nanoseconds, $\left.p_{1}=-10.6 \mathrm{~dB}\right)$ and $(\mathrm{b})$ PIFAs $\left(\tau_{1}=0\right.$ nanoseconds, $p_{1}=0 \mathrm{~dB}$ ): tap 1 of star BS2 configuration, handset free in air, "at the ear."

Test persons' Doppler spectra were generally broader, or smeared out, when compared to those measured free in air, which is reflected in the larger Doppler spread in Table 2. Some influences could be seen in the spectra from shielding by the body or head but the largest influence comes from averaging over ten persons, each walking at a different speed. The effect of different antenna types is comparable to that free in air.

\subsection{Interferer correlations}

The cross-correlations between BS signals for the same antenna branch (interferer correlation) show higher values for the slow fading than for the fast fading, just as with the antenna branch correlations. The interferer correlation coefficients are throughout clearly lower than the branch correlations. Fast fading is barely correlated between BSs and slow fading is only in the new building indoors, and is on average moderately correlated. A histogram of all the interferer coefficients measured in the new building indoors reveals a bimodal distribution as in Figure 11a. Note that two real signals are correlated here. The most probable correlation values, around -0.65 and +0.85 , are actually not so low. Bimodal distributions in the old building were not found for the star BS configuration (Figure 11b), suggesting more 


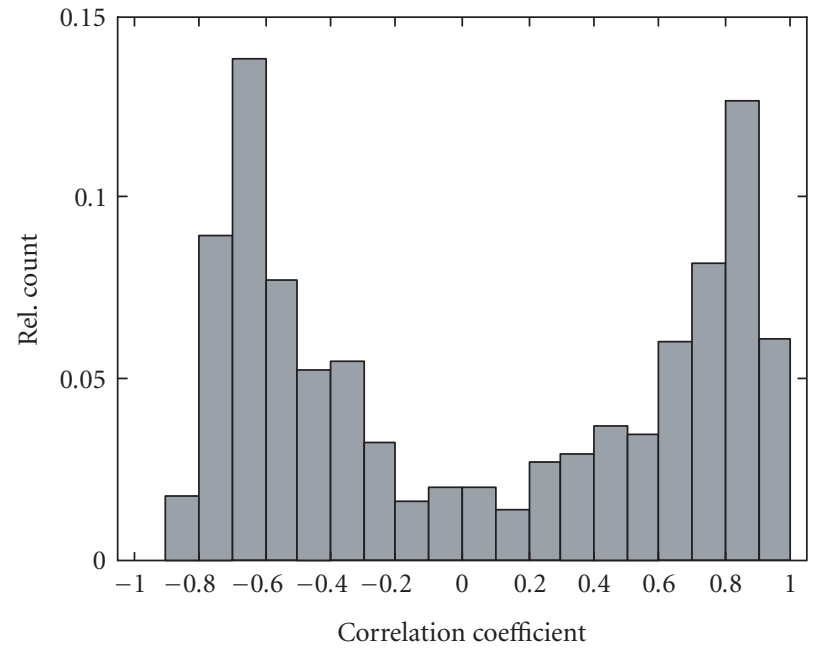

(a)

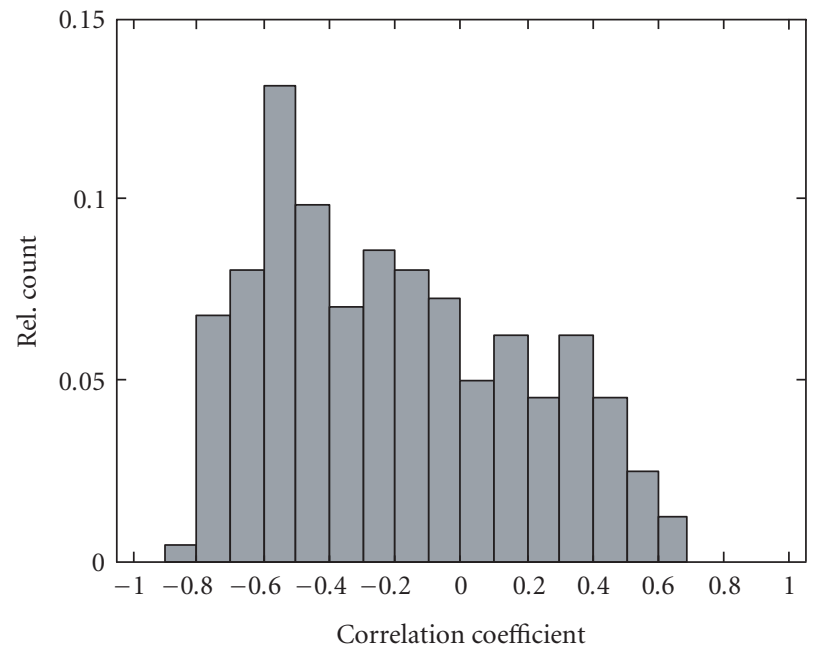

(b)

Figure 11: (a) Histogram of all (real-valued) slow fading interferer correlation coefficients measured in the new building indoors. (b) Values in star BS configuration in the old building indoors.

similar propagation paths for inline than for star. We have no explanation for the fact that most of the coefficients are negative. The fact that in the new building the distribution of correlation coefficients did no strongly depend on the BS configuration hints on guiding as the main propagation mechanism as opposed to penetration in the old building. Effects of guiding in the new building were even suggested by the fast fading correlation. The combination of BSs that were likely to propagate along the same route to the measurement location had on average three to four times higher correlation coefficients than the other two combinations, irrespective of the antenna type. The maximum average value measured was 0.32 for BS1 and BS2, inline with PIFAs.

\subsection{Intertap correlations}

Correlations between tap signals, for the same antenna branch and BS, are low, both in the outdoor-to-indoor and the outdoor cases. The highest average value found was 0.65 . These values confirm the generally assumed uncorrelated scattering for our measurement environments.

\section{CONCLUSIONS}

We measured a number of characteristics that determine the potential diversity gain of multiple antennas on a small handset such as branch correlations, amplitude/power distributions, Doppler spectra, and mean branch power differences. We measured simultaneously on three base stations for three different typical mobile environments: indoor, outdoor-toindoor, and outdoor.

The channel characteristics are generally inline with classical assumptions as regards Rayleigh amplitude distribu- tions and uncorrelated scattering. Doppler spectra, however, are only seldom of classical shape. The branch crosscorrelation values are favourably low, especially for the fast fading, down to very small separations between antennas on a mobile handset if the environment allows. In our outdoor scenario, this was not always the case. Interfering base station signals can show moderate to high correlation values, positive or negative, with respect to their slow fading components under guiding conditions as in one of our indoor environments. A handset design optimised for handling by users should take into account the spread in channel characteristics caused by users and especially should seek a solution to the problem of large mean branch power imbalances between the antennas.

\section{ACKNOWLEDGMENTS}

Nokia is acknowledged for financial and technical support of this work. Patrick Eggers supplied the project with valuable background and possible solutions, as did Morten Jeppesen in the first year of the project. Steen Larsen of the E-værksted was responsible for realisation of the measurement hardware and setting up the campaigns. Istvan Kovács and Devendra Prasad are gratefully acknowledged for their realisation of the data acquisition system software and their support during the campaigns. José Klaus Gonzalez implemented the SAGE software.

\section{REFERENCES}

[1] W. C. Jakes, "New techniques for mobile radio," Bell Laboratory Rec., vol. 48, no. 11, pp. 326-330, 1970.

[2] P. E. Mogensen and J. Wigard, "On antenna- and frequency 
diversity in GSM related systems (GSM-900, DCS-1800, and PCS1900)," in Proc. 7th IEEE International Symposium on Personal, Indoor and Mobile Radio Communications, vol. 3, pp. 1272-1276, Taipei, Taiwan, October 1996.

[3] R. Vaughan and J. B. Andersen, "Antenna diversity in mobile communications," IEEE Trans. Vehicular Technology, vol. 36, no. 4, pp. 149-172, 1987.

[4] H. Arai, S. Hosono, and N. Goto, "A flat diversity antenna by disk loaded monopole and notch array," in Proc. IEEE Antennas and Propagation Society International Symposium, vol. 2, pp. 1085-1088, Chicago, Ill, USA, July 1992.

[5] K. Ogawa and T. Uwano, "A diversity antenna for very small 800-MHz band portable telephones," IEEE Trans. Antennas and Propagation, vol. 42, no. 9, pp. 1342-1345, 1994.

[6] G. F. Pedersen, S. Widell, and T. Ostervall, "Handheld antenna diversity evaluation in a DCS-1800 small cell," in Proc. 8th IEEE International Symposium on Personal, Indoor and Mobile Radio Communications, vol. 2, pp. 584-588, Helsinki, Finland, September 1997.

[7] G. F. Pedersen, J. Ø. Nielsen, K. Olesen, and I. Z. Kovacs, "Antenna diversity on a UMTS handheld phone," in Proc. 10th IEEE International Symposium on Personal, Indoor and Mobile Radio Communications, vol. 1, pp. 152-156, Osaka, Japan, September 1999.

[8] H. Arai, N. Igi, and H. Hanaoka, "Antenna-gain measurement of handheld terminals at $900 \mathrm{MHz}$," IEEE Trans. Vehicular Technology, vol. 46, no. 3, pp. 537-543, 1997.

[9] G. F. Pedersen, J. O. Nielsen, K. Olesen, and I. Z. Kovacs, "Measured variation in performance of handheld antennas for a large number of test persons," in Proc. 48th IEEE Vehicular Technology Conference, vol. 1, pp. 505-509, Ottawa, Ontario, Canada, May 1998.

[10] W. A. Th. Kotterman, D. Prasad, K. Olesen, P. Eggers, I. Z. Kovács, and G. F. Pedersen, "Channel measurement set-up for multi antenna handheld terminal and multiple (interfering) base stations," in Proc. International Symposium on Wireless Personal Multimedia Communications, vol. 1, pp. 153-157, Aalborg, Denmark, September 2001.

[11] W. A. Th. Kotterman, G. F. Pedersen, K. Olesen, and P. Eggers, "Cable-less measurement set-up for wireless handheld terminals," in Proc. 12th IEEE International Symposium on Personal, Indoor and Mobile Radio Communications, vol. 1, pp. B112B116, San Diego, Calif, USA, October 2001.

[12] W. C. Jakes, Ed., Microwave Mobile Communications, John Wiley \& Sons, New York, NY, USA, 1974.

[13] L.-C. Wang, G. L. Stüber, and C.-T. Lea, "Effects of Rician fading and branch correlation on a local-mean-based macrodiversity cellular system," IEEE Trans. Vehicular Technology, vol. 48, no. 2, pp. 429-436, 1999.

[14] J. Zhang and V. Aalo, "Effect of macrodiversity on averageerror probabilities in a Rician fading channel with correlated lognormal shadowing," IEEE Trans. Communications, vol. 49, no. 1, pp. 14-18, 2001.

[15] M.-S. Alouini and M. K. Simon, "Dual diversity over correlated log-normal fading channels," IEEE Trans. Communications, vol. 50, no. 12, pp. 1946-1959, 2002.

[16] M. D. Austin and G. L. Stüber, "Exact cochannel interference analysis for log-normal shadowed Rician fading channels," Electronics Letters, vol. 30, no. 10, pp. 748-749, 1994.

[17] M. Zorzi, "Power control and diversity in mobile radio cellular systems in the presence of Ricean fading and log-normal shadowing," IEEE Trans. Vehicular Technology, vol. 45, no. 2, pp. 373-382, 1996.

[18] M. Pratesi, F. Santucci, F. Graziosi, and M. Ruggieri, "Outage analysis in mobile radio systems with generically correlated log-normal interferers," IEEE Trans. Communications, vol. 48, no. 3, pp. 381-385, 2000.

[19] B. H. Fleury, M. Tschudin, R. Heddergott, D. Dahlhaus, and K. I. Pedersen, "Channel parameter estimation in mobile radio environments using the SAGE algorithm," IEEE JSAC for Wireless Communications Series, vol. 17, no. 3, pp. 434-450, 1999.

[20] W. A. Th. Kotterman, "Testing measured outdoor data on correlation properties between scatterers and between antenna branches of a mobile handset," in Proc. International Symposium on Wireless Personal Multimedia Communications, vol. 1, pp. 176-180, Yokosuka, Japan, October 2003.

[21] R. Vaughan and J. B. Andersen, Eds., Channels, Propagation and Antennas for Mobile Communications, IEE, London, UK, 2003.

[22] S. A. Abbas and A. U. Sheikh, "On understanding the nature of slow fading in LOS microcellular channels," in Proc. 47th IEEE Vehicular Technology Conference, vol. 2, pp. 662666, Phoenix, Ariz, USA, May 1997.

[23] J. B. Andersen, "Statistical distributions in mobile communications using multiple scattering," in Proc. URSI General Assembly, Maastricht, Netherlands, August 2002.

Wim A. Th. Kotterman graduated from Delft University of Technology, the Netherlands, in physics on acoustic wave field theory. He worked for 13 years at KPN Research Labs, Leidschendam, the Netherlands, as a Scientist in the field of radio network planning and radio system optimisation before becoming an Associate Professor at the Department of Communication Technology, Aalborg University, Denmark,

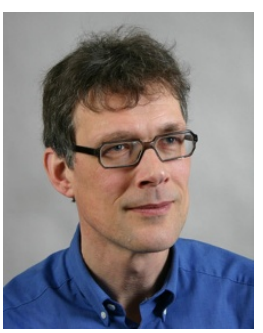
in 2000. His current interests are in channel sounding, the design of channel sounding equipment, and multiantenna handset channel modelling. Wim Kotterman has been a national representative for the Netherlands in the European cooperation projects COST207, COST231, and COST259.

Gert F. Pedersen was born in 1965 . He received the B.S.E.E. degree in electrical engineering from the College of Technology in Dublin, Ireland, and the M.S.E.E. and Ph.D. degrees from Aalborg University in 1993 and 2003, respectively. He has been employed by Aalborg University, Centre for Personkommunikation, since 1993, where he is currently working as an Associate Professor heading the Antenna group. His re-

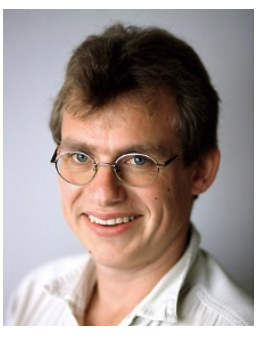
search has focused on radio communication for mobile terminals including small antennas, antenna systems, propagation, and biological effects. He has also worked as a Consultant for the development of antennas for mobile terminals including the first internal antenna for mobile phones in 1994 with very low specific absorption rate (SAR), the first internal triple band antenna in 1998 with low SAR and high efficiency, the smallest internal dual band antenna in 2000, and various antenna diversity systems rated as the most efficient in the market. Recently, he has been involved in establishing a method to measure the communication performance for mobile terminals that can be used as a basis for a $3 \mathrm{G}$ standard, where measurements also including the antenna will be needed. Further, he is involved in small terminals for $4 \mathrm{G}$ including several antennas (MIMO systems) to enhance the data communication. 
Kim Olesen received his Master's degree in electronic engineering from Aalborg University, Denmark, in 1988. From 1988 to 1993, he was employed in private companies, developing analog radio equipment like maritime radios at very high frequency (VHF) and Nordic mobile telephony (NMT) at ultrahigh frequency (UHF). From 1994 onwards, he has been employed at Aalborg University as Head of

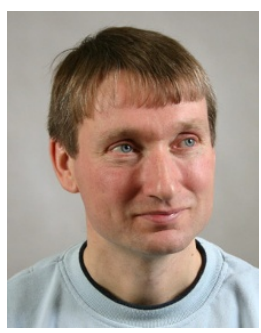
the Electronic Workshop in the Department of Communication Technology, Institute of Electronic Systems. His interests are in the design and construction of measurement systems, mainly for research in the field of antennas and propagation. His design activities range from component level to system level, both analog and digital from $\mathrm{DC}$ to $6 \mathrm{GHz}$. 